\title{
EDITORIAL
}

\section{Intraoperative Transesophageal Echocardiography: A Safeguard for Cardiovascular Surgery!}

\author{
${ }^{1}$ Kathirvel Subramaniam, ${ }^{2}$ Cynthia MQ Wells
}

How to cite this article: Subramaniam K, Wells CMQ. Intraoperative Transesophageal Echocardiography: A Safeguard for Cardiovascular Surgery!. J Perioper Echocardiogr 2014;2(2):49-50.

\section{Source of support: Nil}

Conflict of interest: None

We frequently describe the usefulness of perioperative transesophageal echocardiography as a monitoring and diagnostic device. However, transesophageal echocardiography (TEE) silently performs a third vital role in the primary and secondary prevention of iatrogenic injuries during cardiovascular surgery. Injury to cardiovascular structures can happen during any cardiac, vascular and intrathoracic surgical procedures and also during various cardiovascular percutaneous interventional procedures. Iatrogenic injuries are associated with increased patient morbidity and mortality and intraoperative TEE, if used during these procedures can detect the injuries early so that they can be addressed before the patients leave the operating room (secondary prevention). A delay in the diagnosis to the postoperative period increases the risk of hemodynamic instability and the acuity of presentation during re-exploration operation thereby worsening patient outcomes. For example, detection of a new wall motion abnormality by TEE after mitral valve repair will alert the surgeon of a possible circumflex coronary artery injury. A second pump run and immediate revascularization will salvage the ischemic myocardium. Late diagnosis in the intensive care unit may lead to permanent injury and scarring of the myocardium. TEE also plays a role in the primary prevention by identifying the risk factors for iatrogenic injury and provides a warning signal to the surgeon of what not to do. For example, a cardiothoracic surgeon may be alerted to severe mitral annular calcification with the inherent risk

\footnotetext{
${ }^{1}$ Visiting Associate Professor, ${ }^{2}$ Assistant Professor

1,2Department of Anesthesiology, University of Pittsburgh Pittsburgh, PA, USA
}

Corresponding Author: Kathirvel Subramaniam, Visiting Associate Professor, Department of Anesthesiology University of Pittsburgh, Pittsburgh, PA 15213, USA, e-mail: subramaniamk@upmc.edu of atrioventricular groove injury and left ventricular rupture after mitral valve intervention in such a patient.

In this issue of the Journal of Perioperative Echocardiography, Ravi Raj et al describe an iatrogenic injury to the mitral valve during aortic valve surgery. ${ }^{1} \mathrm{As}$ visualized in the ME long-axis view, the anterior mitral leaflet is in continuity with the aortic valve structure, known as the aortomitral curtain. In reality, the potential for either direct or indirect injury to the anterior mitral leaflet could occur during any surgery on aortic valve. An indirect injury could occur while removing severely calcific speckles from the aortic valve as described by the authors. Subvalvular injuries from suction catheters inserted into left ventricle through the mitral valve have also been described. ${ }^{2}$ With the increasing use of transcatheter aortic valve implantations, there have been reports of valve prostheses protruding low into the ventricular cavity, causing disruption of the mitral valve apparatus. ${ }^{3}$

Similarly, any procedure on the mitral valve has the potential to cause a suture inclusion injury to the cusps of aortic valve, most often affecting the noncoronary and left coronary cusps. ${ }^{4}$ Thus, a high index of suspicion should be maintained following any valve repair or replacement. Post-bypass TEE examination should include not only the valve intervened but also adjacent anatomically closely related valve structures. The detection of a new regurgitant jet in an adjacent valve should alert the echocardiographer to possible injury. A comprehensive post-bypass TEE is essential so as not to miss iatrogenic injuries, especially with the knowledge that new regurgitant jets may be difficult to visualize due to acoustic shadowing from the newly implanted prosthetic material. Transgastric views are particularly useful to rule out these jets, especially aortic incompetence. Several other rare forms of cardiovascular injuries that are rather easily identified by TEE have been reported in the literature including left atrial dissection after mitral valve surgery, ventricular septal defects after septal myomectomy and development of various fistulae, such as left ventricular outflow to right atrial fistula after aortic valve replacement. It is critical to identify such injuries in the operating room in order to avoid bad outcomes. 
In recent years, with the increases in minimally invasive surgical and percutaneous interventional procedures, patients are being exposed to higher risk of iatrogenic injuries. Minimally invasive cardiac procedures (robotic mitral valve procedure), endovascular stenting procedures (aortic endografts), percutaneous valve procedures (mitraclip, transcatheter aortic valve procedures) and cardiac catheterization and electrophysiology laboratory procedures (radiofrequency arrhythmia ablations, atrial appendage closure (Lariat's procedure) and atrial septal defect closure are few of those procedures increasing in popularity. Cardiac structures and vasculature are exposed to guide wires, cannulations, balloons and grafts continuously during these procedures. Instrumentation injuries (vena cava, ventricles, coronary sinus, aorta, atrial septum) and prosthesis migrations (embolization of atrial septal occluders and transcatheter aortic valves) have been described. There is a learning curve associated with these procedures and, during that time, there is the potential for a higher incidence of patient injuries. TEE provides the perfect safeguard as an accompaniment to these procedures by its ability to guide the procedure and continuously monitor for signs of injury.

Aortic injuries are not uncommon and are one of the well-studied iatrogenic injuries in the field of cardiac surgery. In a recent database study from the Society of Thoracic Surgeons, iatrogenic aortic injuries were reported in $0.06 \%$ of cases with an overall mortality rate of $48 \%$ for those affected. ${ }^{5}$ This mortality is higher than naturally presenting aortic dissections, because these patients face the combined risk of a required cardiac surgical procedure and an additional aortic procedure when injury occurs. Factors influencing higher mortality include the need for urgent alternate cannulation, bleeding from the aortic injury with hemodynamic instability, and the requirement for longer period of myocardial preservation. Iatrogenic aortic dissections are most often associated with femoral cannulations, preoperative steroid therapy, peripheral vascular disease, Asian race and age more than 60 years in their database. Routine TEE-guided cannulation and a full aortic examination after instrumentation (cannulation, decannulation, intraaortic balloon pump insertion and endovascular procedures) allow for early identification of aortic dissections and prior to the onset of hemodynamic instability. Such large database studies are vital to identify the risk factors for all forms of cardiac iatrogenic injuries, as they are rare and underreported but are associated with high mortality. TEE for sure plays a perfect safeguard for patients in the cardiovascular operating rooms!

\section{REFERENCES}

1. Raj R, Mathew S, Puri GD. Iatrogenic mitral valve perforation following CABG and aortic valve replacement: a rare complication detected by post-bypass transesophageal echocardiography. J Periop Echocardiogr 2014;2(2):75-76.

2. Steiman J, Chaney MA. Iatrogenic mitral valve injury during aortic valve replacement: importance of intraoperative transesophageal echocardiography. J Cardiothorac Vasc Anesth 2011;25(3):586.

3. Wong DR, Boone RH, Thompson CR, et al. Mitral valve injury late after transcatheter aortic valve implantation. J Thorac Cardiovasc Surg 2009;137(6):1547-1549.

4. Santiago M1, El-Dayem MA, Dimitrova G, Awad H. Missed diagnosis of iatrogenic acute aortic insufficiency after mitral valve surgery. Int Anesthesiol Clin 2011;49:26-31.

5. Williams ML, Sheng S, Gammie JS, et al. Aortic dissection as a complication of cardiac surgery: report from the Society of Thoracic Surgeons database. Ann Thorac Surg 2010;90(6):18121816. Discussion 1816-1817. 